PROCEEDINGS OF THE

AMERICAN MATHEMATICAL SOCIETY

Volume 127, Number 7, Pages 2147-2154

S 0002-9939(99)04892-3

Article electronically published on March 3, 1999

\title{
FLUX FOR MEAN CURVATURE 1 SURFACES IN HYPERBOLIC 3-SPACE, AND APPLICATIONS
}

\author{
WAYNE ROSSMAN, MASAAKI UMEHARA, AND KOTARO YAMADA
}

(Communicated by Peter Li)

\begin{abstract}
Using the Bryant representation, we define a flux on homology classes of CMC-1 surfaces in $\mathcal{H}^{3}$, satisfying a balancing formula which is useful to show nonexistence of certain kinds of complete CMC-1 surfaces.
\end{abstract}

\section{INTRODUCTION}

Recently, flux and balancing formulas have been found very useful techniques for proving results about surfaces of constant mean curvature $H$ (CMC- $H$ ) in 3dimensional space forms. For CMC surfaces in Euclidean space $\mathbf{R}^{3}$ a flux has been defined on homology classes of closed curves $\gamma$ within the surfaces [KKS], which we call the KKS-flux here. Also, in [KKMS], a corresponding flux is defined for CMC surfaces in hyperbolic space $\mathcal{H}^{3}$ of constant sectional curvature -1 , which we call the KKMS-flux.

In this work we define a flux $F l$ using the Bryant representation [Bry]. $F l$ satisfies a balancing formula (Theorem 1 ). We give a condition for when a regular embedded end has nonzero $\mathrm{Fl}$ flux (Corollary 5), which is useful to show nonexistence of complete CMC-1 surfaces under certain assumptions about the behavior of their ends (Corollary 3 and Theorem 7).

This new definition of flux for CMC- 1 surfaces in $\mathcal{H}^{3}$ is motivated by the KKSflux and KKMS-flux. The KKS-flux (resp. KKMS-flux) is the sum of an integral along a curve $\gamma$ in the surface and an integral over a "cap" which is some 2-dimensional disk in $\mathbf{R}^{3}$ (resp. $\mathcal{H}^{3}$ ) bounded by $\gamma$. When $H=0$, the integral over the cap automatically vanishes. When $H \neq 0$ (resp. $|H|>1$ ) and the surface is of finite topology in $\mathbf{R}^{3}$ (resp. $\mathcal{H}^{3}$ ), the properly embedded annular ends of the surface converge asymptotically to Delaunay surfaces [KKS] (resp. hyperbolic Delaunay surfaces [KKMS]); hence the curves in neighborhoods of the ends can be kept at a uniformly bounded length, and the caps bounded by these curves can be kept to a uniformly bounded area. These properties are useful for computing the KKS-flux (resp. KKMS-flux) when $|H|>0$ (resp. $|H|>1$ ).

However, for a minimal surface in $\mathbf{R}^{3}$ (resp. CMC- $H$ surface in $\mathcal{H}^{3}$ ) with $H=0$ (resp. $|H| \leq 1$ ), the curve $\gamma$ has arbitrarily large length and the cap has arbitrarily large area at an end. This means that it can be difficult to compute the integrals in

Received by the editors October 15, 1997.

1991 Mathematics Subject Classification. Primary 53A10; Secondary 53A35, 53A42.

The authors were supported by Volkswagen-Stiftung (RiP Program in Mathematisches Forschungsinstitut Oberwolfach). The third author was supported by the Inamori Foundation.

(C)1999 American Mathematical Society 
the definition of KKS-flux (resp. KKMS-flux), in particular, for complete minimal surfaces in $\mathbf{R}^{3}$ (resp. CMC-1 surfaces in $\mathcal{H}^{3}$ ). For minimal surfaces in $\mathbf{R}^{3}$ one can use the Weierstrass representation to give an alternate definition of KKS-flux that helps us compute the integrals (see Remark after Theorem 1). The authors initially defined $F l$ in the hopes of using the Bryant representation in an analogous way to compute the KKMS-flux for CMC-1 surfaces in $\mathcal{H}^{3}$.

However, due to complications that occur in the $\mathcal{H}^{3}$ case, the authors could not determine the equivalence of $F l$ flux and KKMS-flux. There are indications of equivalence: the KKS-flux can be considered as a limit of $F l$ flux (Remark after Theorem 1$)$, and both fluxes are $\mathfrak{s l}(2, \mathbf{C})$-valued, and there are other common properties (Example 8). Even with this question remaining open, however, the authors found useful applications for the $F l$ flux.

\section{A FluX FORMUla FOR CMC-1 IMMERSIONS}

Let $M$ be a Riemann surface. We introduce the flux $F l$ for any conformal branched CMC-1 immersion $x: M \rightarrow \mathcal{H}^{3}$. (A point where the first fundamental form vanishes is called a branch point of a CMC-1 surface. Branch points on CMC1 surfaces are all isolated.) Let $\widetilde{M}$ be the universal cover of $M$. Take Weierstrass data $(g, \omega)$ of $x$ and let $F: \widetilde{M} \rightarrow P S L(2, \mathbf{C})$ be the unique lift of $x$ with respect to $(g, \omega)$, which is a null holomorphic map (cf. [UY3, Theorem 1.4]). Let $G$ and $Q$ be the hyperbolic Gauss map and the Hopf differential of $x$.

The inverse matrix function, $F^{-1}: \widetilde{M} \rightarrow P S L(2, \mathbf{C})$, is also a null holomorphic map, and a new "dual" branched CMC-1 immersion $x^{\#}: \widetilde{M} \rightarrow \mathcal{H}^{3}$ is determined by $x^{\#}:=F^{-1} \cdot\left(F^{-1}\right)^{*}$. As shown in [UY5], this duality operation interchanges the roles of the hyperbolic Gauss map and the secondary Gauss map. The Weierstrass data of the dual surface is given by $\left(G, \omega^{\#}\right)$, where

$$
\omega^{\#}:=-Q / d G \text {. }
$$

In particular, the following identity holds:

$$
\alpha^{\#}:=\left(F^{-1}\right)^{-1} \cdot d\left(F^{-1}\right)=-d F \cdot F^{-1}=\left(\begin{array}{cc}
G & -G^{2} \\
1 & -G
\end{array}\right) \omega^{\#} .
$$

The key point is that $G$ and $Q$ are single-valued on $M$, and thus $\alpha^{\#}$ is singlevalued on $M$. So we can define a well-defined $\mathfrak{s l}(2, \mathbf{C})$-valued flux $F l(\gamma)$ for any loop $\gamma$ on $M$ by

$$
F l(\gamma):=\frac{-1}{2 \pi i} \int_{\gamma} d F \cdot F^{-1} .
$$

Since $d F \cdot F^{-1}$ is holomorphic on $M$, the flux $F l(\gamma)$ depends only on the homology class represented by the loop $\gamma$.

Now we suppose that there exist a closed Riemann surface $\bar{M}$ and a finite number of points $\left\{p_{1}, \ldots, p_{n}\right\}$ such that $M$ is biholomorphic to $\bar{M} \backslash\left\{p_{1}, \ldots, p_{n}\right\}$ and the first fundamental form is complete at each $p_{j}$. Each $p_{j}$ is called an end of the branched surface. (These conditions are automatically satisfied when $x$ is an immersion which is complete and of finite total curvature.) Then we have a well-defined $\mathfrak{s l}(2, \mathbf{C})$-valued flux $F l_{j}$ at each end $p_{j}$ by

$$
F l_{j}:=F l\left(\gamma_{j}\right) \quad\left(=\frac{-1}{2 \pi i} \int_{\gamma_{j}} d F \cdot F^{-1}\right),
$$


where $\gamma_{j}$ is a loop surrounding the end $p_{j}$. The following balancing formula is easily proved by triangulating $\bar{M}$ and summing the results of the Cauchy residue theorem.

Theorem 1. Let $x: M \rightarrow \mathcal{H}^{3}$ be a branched CMC-1 immersion. Then the total sum of flux at the ends vanishes; that is, we have the balancing formula

$$
\sum_{j=1}^{n} F l_{j}=0
$$

Remark. For a minimal immersion $x: \bar{M} \backslash\left\{p_{1}, \ldots, p_{n}\right\} \rightarrow \mathbf{R}^{3}$ with the Weierstrass data $(g, \omega)$, the KKS-flux is given by the residue of the meromorphic form $\left(\left(1-g^{2}\right), \sqrt{-1}\left(1+g^{2}\right), 2 g\right) \omega$ at each end. As seen in [UY2], the Weierstrass representation can be considered as a limit of the Bryant representation. Using this, one can easily see that KKS-flux is the limit of the flux $F l$.

Next we consider one regular end of a CMC-1 immersion. Using a local complex coordinate $(U, z)$ around an end $z=0$, the Hopf differential $Q$ is expressed as

$$
Q(z)=q(z) d z^{2}=\left(\frac{q_{-2}}{z^{2}}+\frac{q_{-1}}{z}+\cdots\right) d z^{2} .
$$

An end $z=0$ is called a regular end of Type $I$ if $q_{-2} \neq 0$, and called a regular end of Type II if $q_{-2}=0$. (Regular ends are those at which the hyperbolic Gauss map is meromorphic. An end is regular if and only if the Hopf differential $Q$ is either holomorphic or has a pole of order at most 2 at the end. [Bry], [UY1].)

Regular ends of Type I. We compute the flux at an end $x$ of Type I. We set $z=0$ at the end. Then the Weiestrass data $\left(G, \omega^{\#}\right)$ of the dual end $x^{\#}$ can be normalized as

$$
G(z)=z^{l} \hat{G}(z), \quad \omega^{\#}=z^{k} \hat{w}^{\#}(z) d z \quad\left(\hat{w}^{\#}(0) \neq 0, \hat{G}(0) \neq 0\right),
$$

where $l>0$ and $k$ are integers, and $\hat{G}(z)$ and $\hat{w}^{\#}(z)$ are holomorphic functions. Since the metric of $x^{\#}$ is also complete (see [Y] or [UY5]), by [UY1] we have $k+1<0$. Since $q_{-2} \neq 0,(1)$ yields that $k+l=-1$. So the diagonal components $\pm G \omega^{\#}$ of $\alpha^{\#}=-d F \cdot F^{-1}$ have simple poles at $z=0$, and hence:

Proposition 2. A regular end of Type I has a non-vanishing flux.

Corollary 3. There is no branched 1-ended CMC-1 surface with Type I end.

Regular ends of Type II. Next we compute the flux for CMC-1 ends of Type II. Since this is not as easy as the Type I case, we do it only for some special cases, including the case of embedded ends. For the case of minimal surfaces in $\mathbf{R}^{3}$, any regular end of Type II has vanishing flux, but this is not true for the CMC-1 case in $\mathcal{H}^{3}$ (see Example 6).

We fix a regular end of Type II at $z=0$. The Weierstrass data $\left(G, \omega^{\#}\right)$ of the dual end $x^{\#}$ can again be normalized with integers $l(l>0)$ and $k$ as

$$
G(z)=z^{l} \hat{G}(z), \quad \omega^{\#}=z^{k} \hat{w}^{\#}(z) d z \quad\left(\hat{w}^{\#}(0) \neq 0, \hat{G}(0) \neq 0\right) .
$$

Since $q_{-2}=0$, Lemma 2 and Proposition 4 in [UY5] yield that the secondary Gauss map $g$ is of the form $g(z)=z^{l} \hat{g}(z)$, where $\hat{g}(z)$ is a holomorphic function satisfying $\hat{g}(0) \neq 0$. Since $g$ is then single-valued at $z=0, x^{\#}$ is also single-valued. (When $g$ and $G$ are both single-valued on $U$, then so are the lifts $F$ and $F^{-1}$ of $x$ and $x^{\#}$. 
See section 3 of [Sm] or Theorem 1.6 of [UY3].) Hence by Theorem 2.4 in [UY1], the log-term coefficient $\theta$ of the following differential equation vanishes:

$$
X^{\prime \prime}-\frac{\left(z^{k} \hat{w}^{\#}(z)\right)^{\prime}}{z^{k} \hat{w}^{\#}(z)} X^{\prime}+q(z) X=0,
$$

where $Q=q(z) d z^{2}$. We set

$$
\hat{w}^{\#}(z)=w_{0}+w_{1} z+w_{2} z^{2}+\cdots,
$$

where $w_{0} \neq 0$. By the expressions (6) and (1), we have

$$
w_{0}=-\frac{q_{l+k-1}}{l \hat{G}(0)}, \quad w_{1}=-\frac{q_{l+k}+(l+1) w_{0} \hat{G}^{\prime}(0)}{l \hat{G}(0)} .
$$

Let $\gamma$ be a loop surrounding the origin. Since $q_{-2}=0, G \omega^{\#}=-Q \cdot(G / d G)$ and $G^{2} \omega^{\#}=-G Q \cdot(G / d G)$ are holomorphic at $z=0$. So, we have

$$
\frac{-1}{2 \pi i} \int_{\gamma}\left(\begin{array}{cc}
G & -G^{2} \\
1 & -G
\end{array}\right) \omega^{\#}=-\left(\begin{array}{cc}
0 & 0 \\
w_{m} & 0
\end{array}\right),
$$

where $m=-k-1$ is the multiplicity of the end (see [UY1]). It can be easily seen that equation (9) has no log-term if and only if (see [Bie])

$$
\begin{aligned}
w_{1} & =-q_{-1} w_{0} & & (m=1), \\
4 w_{2} & =-q_{0} w_{0}-3 q_{-1} w_{1}-q_{-1}^{2} w_{0} & & (m=2) .
\end{aligned}
$$

By (1), we have $\operatorname{ord}_{z=0} Q=l+k-1 \geq-1$, where equality holds if and only if $q_{-1} \neq 0$. Then by (10) and (11) and the above conditions, we have:

Theorem 4. A regular Type II end of multiplicity $m(=1$ or 2$)$ has non-vanishing flux if and only if

$$
\begin{aligned}
& q_{-1} \neq 0 \\
& (m=1), \\
& q_{-1}=0, q_{0} \neq 0 \quad \text { or } \\
& q_{-1} \neq 0, \quad 4\left(q_{0}-\frac{\hat{G}^{\prime}(0)}{\hat{G}(0)} q_{-1}\right)+q_{-1}^{2} \neq 0 \quad(m=2) .
\end{aligned}
$$

Since an end is embedded if and only if $m=1$, by Proposition 2 and Theorem 4, we have:

Corollary 5. A regular embedded end has vanishing flux if and only if the Hopf differential $Q$ is holomorphic at the end.

Example 6. We note that the flux does not depend only on the asymptotic behavior of the end even in the Type I case. For example, the catenoid cousin has the following data defined on $M=\mathbf{C} \backslash\{0\}$ :

$$
g=z^{\mu}, \quad G=z, \quad Q=\frac{1-\mu^{2}}{4} \frac{d z^{2}}{z^{2}} \quad\left(\mu \in \mathbf{R}^{+} \backslash\{1\}\right) .
$$

The flux at $z=0$ is then given by

$$
\frac{-1}{2 \pi i} \int \frac{1-\mu^{2}}{4}\left(\begin{array}{ll}
z^{-1} & -1 \\
z^{-2} & -z^{-1}
\end{array}\right) d z=\frac{\mu^{2}-1}{4}\left(\begin{array}{rr}
1 & 0 \\
0 & -1
\end{array}\right) .
$$

Now we consider another example given by

$$
g=z^{\mu}, \quad G=z+\frac{1}{2} z^{2}, \quad Q=\frac{1}{2}(S(g)-S(G))=\frac{1-\mu^{2}}{4} \frac{1}{z^{2}}-\frac{3}{4} \frac{1}{(1+z)^{2}} .
$$


( $S$ is the Schwarzian derivative [Bry], [UY1].) This example is a branched surface (cf. [UY3, Theorem 2.2]). Since $G(0)=0$, one can check using (5.16) of [UY1] that the end at the origin is asymptotic to the end at $z=0$ of the above catenoid cousin. The flux at the origin is

$$
\frac{-1}{2 \pi i} \int \frac{1-\mu^{2}}{4}\left(\begin{array}{cc}
z^{-1} & 0 \\
-z^{-1} & -z^{-1}
\end{array}\right) d z=\frac{\mu^{2}-1}{4}\left(\begin{array}{rr}
1 & 0 \\
-1 & -1
\end{array}\right) .
$$

Hence this is different from the flux of the catenoid cousin.

Remark. As discussed in Theorem 3.2 of [RUY1], branched CMC-1 immersions $x$ are divided into the following three classes:

$$
\text { - irreducible, } \quad \mathcal{H}^{1} \text {-reducible, } \quad \bullet \mathcal{H}^{3} \text {-reducible. }
$$

If the surface $x$ is $\mathcal{H}^{3}$-reducible, then the lift $F$ itself is single-valued; hence the integral $(-1 / 2 \pi i) \int F^{-1} \cdot d F$ on any homology class is well-defined. We call this flux $F l^{\#}$, and it also satisfies a balancing formula on the ends. (It is, in fact, the $F l$ flux for $x^{\#}$. Unlike the $F l$ flux, $F l^{\#}$ does depend on the choice of Weierstrass data.) In the case that $x$ is $\mathcal{H}^{1}$-reducible, we cannot define $F l^{\#}$, but there exists a special Weierstrass data $(g, \omega)$ such that $g \omega$ is single-valued, as seen in [UY1]. So we can define a flux $f l^{\#}$ on homology classes $\gamma$ by $f l^{\#}(\gamma)=(-1 / 2 \pi i) \int_{\gamma} g \omega$. When $x$ is $\mathcal{H}^{3}$-reducible, $f l^{\#}$ is just one of the diagonal components of $F l^{\#}$. The computation of these new fluxes at irregular ends is easier than that of the flux $F l$, because $F^{-1} \cdot d F$ has at most a pole whereas $d F \cdot F^{-1}$ has an essential singularity.

As an application of the flux formula, we give the following result:

Theorem 7. Let $\bar{M}$ be a closed Riemann surface and consider a branched CMC-1 immersion $x: \bar{M} \backslash\left\{p_{1}, \ldots, p_{n}\right\} \rightarrow \mathcal{H}^{3}$ whose ends are all regular. Suppose that $p_{1}$ is a Type I end. Suppose also that the ends $\left\{p_{1}, \ldots, p_{n}\right\}$ are all poles or zeros of the hyperbolic Gauss map $G$. Then $x$ has at least one more Type I end $z=p_{j}$ $(2 \leq j \leq n)$.

Catenoid cousins, 3-ended and 4-ended Costa cousins constructed in [RUY1] and $[\mathrm{N}]$ are examples satisfying the assumptions of the theorem. The assumption that the ends are all poles or zeros of the hyperbolic Gauss map is essential in this theorem (see the following Example 8).

Proof of Theorem \%. Consider the 1-form $\eta:=G \cdot \omega^{\#}$, where $\omega^{\#}:=-Q / d G$. Since all ends of $x$ are regular, $\eta$ is a meromorphic 1-form on $\bar{M}$. Moreover, $\eta$ and $-\eta$ are the diagonal components of the $\mathfrak{s l}(2, \mathbf{C})$-valued 1 -form $-d F \cdot F^{-1}$, so $\eta$ is holomorphic on $\bar{M} \backslash\left\{p_{1}, \ldots, p_{n}\right\}$. Thus we have $\sum_{j=1}^{n} \eta_{j}=0$, where $\eta_{j}$ is the residue of $\eta$ at $p_{j}$. Suppose the Hopf differential $Q$ has at most poles of order 1 at $p_{2}, \ldots, p_{n}$. Then $Q$ has the following Laurent expansions at the ends:

$$
Q(z)=\left(\frac{q_{-2}^{(j)}}{\left(z-p_{j}\right)^{2}}+\frac{q_{-1}^{(j)}}{z-p_{j}}+\cdots\right) d z^{2} \quad(j=1, \ldots, n),
$$

where $q_{-2}^{(1)} \neq 0$ and $q_{-2}^{(j)}=0$ for $j=2, \ldots, n$. Since each end $z=p_{j}(j=1, \ldots, n)$ is a pole or a zero of $G$, we have

$$
G / d G:=a_{j}\left(z-p_{j}\right)+b_{j}\left(z-p_{j}\right)^{2}+\cdots \quad(j=1, \ldots, n),
$$

where $a_{j}$ is a non-zero complex number. Thus we can conclude that $\eta_{1}=-a_{1} q_{-2}^{(1)}$ $(\neq 0)$ and $\eta_{j}(z)=0$ for $j=2, \ldots, n$. This contradicts the relation $\sum_{j=1}^{n} \eta_{j}=0$. 
Example 8. It can be easily seen that there is a complete CMC-1 immersion $x: \mathbf{C} \backslash\{0,1\} \rightarrow \mathcal{H}^{3}$ such that

$$
G=\frac{z+1}{(z-1)^{3}}\left(z^{2}-4 z+1\right), \quad g=z^{2}, \quad Q=\frac{2}{z(z-1)^{2}} d z^{2},
$$

which has three regular ends and only $z=1$ is an end of Type I. This shows that the condition $\left\{p_{1}, \ldots, p_{n}\right\}$ are all poles or zeros of $G$ is essential in Theorem 7 . Moreover, it shows that, just like the flux $F l$, the KKMS-flux cannot always vanish on Type II ends. This is because the KKMS-flux satisfies a balancing formula, and the KKMS-flux for regular ends of Type I does not vanish. (For minimal surfaces in $\mathbf{R}^{3}$, the KKS-flux vanishes for ends of Type II.) This lends further support to conjecture that the $\mathrm{Fl}$ flux and KKMS-flux are equivalent.

Remark. In fact, as there are CMC- $c$ surfaces in $\mathcal{H}^{3}\left(-c^{2}\right)$ which converge to minimal surfaces in $\mathbf{R}^{3}$ as $c \rightarrow 0$ [UY2], we can see that KKMS flux splits into a KKS flux part and a torque part (torque is defined in $[\mathrm{KK}]$ ) as $c \rightarrow 0$. Since torque on a complete embedded regular minimal end of finite total curvature in $\mathbf{R}^{3}$ is nonzero if and only if ord $Q<0$ at the end, it is not surprizing that KKMS-flux for Type II ends in $\mathcal{H}^{3}$ with ord $Q<0$ is nonzero. This behavior is precisely the same as $\mathrm{Fl}$ flux (Corollary 5), so we are led to conjecture that $\mathrm{Fl}$ flux and KKMS-flux are equivalent.

Further remarks. Let $\operatorname{Met}_{1}(\bar{M})$ be the set of positive semi-definite conformal metrics of constant curvature 1 with conical singularities on a closed Riemann surface $\bar{M}$. Suppose that $d u^{2} \in \operatorname{Met}_{1}(\bar{M})$ has conical singularities at points $z_{j} \in \bar{M}$ $(j=1, \ldots, n)$ with order $\beta_{j}(>-1)$, that is, the metric admits a tangent cone of angle $2 \pi\left(\beta_{j}+1\right)>0$ at each $z_{j}$. Following Troyanov [T1], we call $\beta=\sum_{j=1}^{n} \beta_{j} z_{j}$ the divisor represented by $d u^{2}$. As shown in [UY3], there is a canonical correspondence between pseudo-metrics in $\operatorname{Met}_{1}(\bar{M})$ and (not totally umbilic) branched CMC-1 surfaces in $\mathcal{H}^{3}$ with prescribed hyperbolic Gauss map on $\bar{M}$. Our first motivation to formulate Theorem 7 was based on this problem:

Problem. Let $G$ be a meromorphic function on a closed Riemann surface $\bar{M}$ with the ramification divisor

$$
\beta_{0}=m_{1} p_{1}+\cdots+m_{n} p_{n} \quad\left(n, m_{1}, \ldots, m_{n} \in \mathbf{Z}^{+}\right),
$$

where $p_{1}, \ldots, p_{n} \in \bar{M}$ are branch points of $G$. Can one show the non-existence of conformal metrics $d \sigma_{t}^{2} \in \operatorname{Met}_{1}(\bar{M})$ with the divisor

$$
\beta_{t}=\left(t+m_{1}\right) p_{1}+\cdots+m_{n} p_{n}
$$

for $t \notin \mathbf{Z}$ ?

We cannot remove the assumption $t \notin \mathbf{Z}$. In fact, the two pull-backs of the Fubini-Study metric $G^{*} d \sigma_{0}^{2}$ and $g^{*} d \sigma_{0}^{2}$ in Example 6 have the divisors $1 p_{0}+2 p_{1}+$ $1 p_{\infty}$ and $1 p_{0}+1 p_{\infty}$, respectively. When $\bar{M}=P^{1}(\mathbf{C})$, a much stronger assertion is true: there are no metrics $d \sigma_{t}^{2}$ with the divisor $\beta_{t}(t \notin \mathbf{Z})$ even when $\beta_{0}$ is not the ramification divisor of some meromorphic function. However, on a closed surface $\bar{M}$ of genus $\geq 1$, there exists a metric in $\operatorname{Met}_{1}(\bar{M})$ with only one conical singularity, by a result in [T2]. This problem seems to be related with flux formulas for minimal surfaces in $\mathbf{R}^{3}$ and for CMC-1 surfaces in $\mathcal{H}^{3}$ by the following two observations: 
Observation 1. There is no smooth deformation of metrics $d u_{t}^{2} \in \operatorname{Met}_{1}(\bar{M})$ for $t \in \mathbf{R}$ close to 0 such that

(1) $d u_{0}^{2}=G^{*} d \sigma_{0}^{2}$, where $d \sigma_{0}^{2}$ is the Fubini-Study metric on $P^{1}(\mathbf{C})$.

(2) Each $d u_{t}^{2}$ has the divisor $\beta_{t}$.

Observation 2. If the assertion of Theorem 7 would have held without assuming that $\left\{p_{1}, \ldots, p_{n}\right\}$ are all poles or zeros of $G$, then there would be no such metrics as in the problem above.

Proof. As seen in [UY3, p. 211], the difference of Schwarzian derivatives

$$
Q_{t}(z):=\frac{\widetilde{S}\left(d u_{t}^{2}\right)-\widetilde{S}\left(d u_{0}^{2}\right)}{2 t}=\frac{1}{2}\left(\int_{0}^{1} \frac{\partial h_{t s}(z)}{\partial t} d s\right) d z^{2}
$$

is a well-defined, holomorphic 2-differential on $\bar{M} \backslash\left\{p_{1}, \ldots, p_{n}\right\}$, where $\tilde{S}\left(d u_{r}^{2}\right)=$ $h_{r}(z) d z^{2}$ for $r \in \mathbf{R}$. Then by [T1], the limit $Q_{0}:=\lim _{t \rightarrow 0} Q_{t}$ has a pole of order 2 at $p_{1}$ and could possibly have poles of order at most one at $p_{2}, \ldots, p_{n}$. On the other hand, there exists a unique branched CMC-1 surface $x_{t}: \bar{M} \backslash\left\{p_{1}, \ldots, p_{n}\right\} \rightarrow$ $\mathcal{H}^{3}\left(-t^{2}\right)$ with the hyperbolic Gauss map $G$ and with the associated metric $d u_{t}^{2}$ for each $t$ (see [UY3, Theorem 2.2]). We identify the hyperbolic space $\mathcal{H}^{3}\left(-t^{2}\right)$ of constant curvature $-t^{2}$ with the Poincaré ball

$$
\left(\left\{x \in \mathbf{R}^{3}|| x \mid<2 / t\right\}, \frac{4}{4-t^{2}|x|^{2}}|d x|^{2}\right) .
$$

Then $x_{0}=\lim _{t \rightarrow 0} x_{t}: M^{2} \rightarrow \mathbf{R}^{3}$ is a conformal branched immersion with Hopf differential $Q_{0}$ and Gauss map $G$ (see [UY2]). $p_{1}$ is the only Type I end of the immersion $x_{t}(t \in \mathbf{R})$. This makes a contradiction when $t=0$, since the other ends of $x_{0}$ have vanishing flux, since a minimal surface has vanishing flux at Type II ends. This proves Observation 1.

Next we suppose the assertion of Theorem 7 holds without assuming that $\left\{p_{1}, \ldots, p_{n}\right\}$ are all poles or zeros of $G$. Then we have the same contradiction for $x_{t}$ for each fixed $t \notin \mathbf{Z}$, which proves the second observation.

Unfortunately, as seen in Example 6, Theorem 7 fails to hold without assuming that $\left\{p_{1}, \ldots, p_{n}\right\}$ are all poles or zeros of $G$. However, the two observations suggest that there are connections between the set $\operatorname{Met}_{1}(\bar{M})$ and the flux formula on branched conformal CMC-1 immersions. Several related topics are discussed in [RUY2] and [UY6].

\section{ACKNOWLEDGEMENTS}

We wish to thank Shin Kato for fruitful discussions during our stay at Oberwolfach Institute. We also wish to thank L-F. Cheung for his helpful comments and encouragement. We are deeply grateful to the RiP program at Oberwolfach Institute for its kind hospitality and exemplary working conditions.

\section{REFERENCES}

[Bie] L. Bieberbach, Theorie der gewöhnlichen Differentialgleichungen, Zweite Auflage, Springer-Verlag, 1965. MR 83a:34001

[Bry] R. Bryant, Surfaces of mean curvature one in hyperbolic space, Astérisque 154-155 (1987), 321-347. CMP 20:16

[KK] N. Korevaar and R. Kusner, The global structure of constant mean curvature surfaces, Invent. Math. 114 (1993), 311-332. 
[KKS] N. Korevaar, R. Kusner and B. Solomon, The structure of complete embedded surfaces with constant mean curvature, J. Differential Geometry 30 (1989), 465-503. MR 90g:53011

[KKMS] N. Korevaar, R. Kusner, W. H. Meeks and B. Solomon, Surfaces in Hyperbolic space, Amer. J. Math. 114 (1992), 1-43. MR 92k:53116

[N] V. F. de Sousa Neto, Costa type surfaces in hyperbolic space, to appear in the Anais da Academia Brasileira de Matemática.

[RUY1] W. Rossman, M. Umehara and K. Yamada, Irreducible constant mean curvature 1 surfaces in hyperbolic space with positive genus, Tôhoku Math. J. 49 (1997), 449-484. CMP 98:03

[RUY2] - Metrics with conical singularities and CMC-1 surfaces of low total curvature in hyperbolic space, in preparation.

[Sm] A. J. Small, Surfaces of Constant Mean Curvature 1 in $H^{3}$ and Algebraic Curves on a Quadric, Proc. Amer. Math. Soc. 122 (1994), 1211-1220. MR 95b:53011

[T1] M. Troyanov, Metric of constant curvature on a sphere with two conical singularities, in "Differential Geometry", Lect. Notes in Math. vol. 1410, Springer-Verlag, (1989), 296-306. MR 90m:53057

[T2] - Prescribing curvature on compact surfaces with conical singularities, Trans. Amer. Math. Soc. 324 (1991), 793-821. MR 91h:53059

[UY1] M. Umehara and K. Yamada, Complete surfaces of constant mean curvature-1 in the hyperbolic 3-space, Ann. of Math. 137 (1993), 611-638. MR 94c:53015

[UY2] - A parametrization of Weierstrass formulae and perturbation of some complete minimal surfaces of $\mathbf{R}^{3}$ into the hyperbolic 3-space, J. reine u. angew. Math. 432 (1992), 93-116. MR 94e:54004

[UY3] Surfaces of constant mean curvature-c in $H^{3}\left(-c^{2}\right)$ with prescribed hyperbolic Gauss map, Math. Ann. 304 (1996), 203-224. MR 97b:53017

[UY4] Another construction of a CMC-1 surface in $H^{3}$, Kyungpook Math. J. 35 (1996), 831-849.

[UY5] A duality on CMC-1 surface in the hyperbolic 3-space and a hyperbolic analogue of the Osserman Inequality, Tsukuba J. Math. 21 (1997), 229-237. CMP 97:17

[UY6] - Metric of constant curvature one with three conical singularities on 2-sphere, preprint (math.DG/9801137).

[Y] Z. Yu, Value distribution of hyperbolic Gauss maps, Proc. Amer. Math. Soc. 125 (1997), 2997-3001. MR 97m:53016

Graduate School of Mathematics, Kyushu University, Fukuoka 812-8581 Japan

E-mail address: wayne@math.kyushu-u.ac.jp

Current address: Department of Mathematics, Faculty of Science, Kobe University, Rokko, Kobe 657-8501, Japan

E-mail address: wayne@math.kobe-u.ac.jp

Department of Mathematics, Graduate School of Science, Osaka University, ToyONAKA, OSAKA 560-0043 JAPAN

E-mail address: umehara@math.wani.osaka-u.ac.jp

Current address: Department of Mathematics, Faculty of Science, Hiroshima University, Higashi-Hiroshima 739-8526, Japan

E-mail address: umehara@math.sci.hiroshima-u.ac.jp

Department of Mathematics, Faculty of Science, Kumamoto University, Kumamoto 860-8555 JAPAN

E-mail address: kotaro@gpo.kumamoto-u.ac.jp 